\title{
Solving a System of Linear Volterra Integral Equations Using the Modified Reproducing Kernel Method
}

\author{
Li-Hong Yang, Hong-Ying Li, and Jing-Ran Wang \\ College of Science, Harbin Engineering University, Heilongjiang 150001, China \\ Correspondence should be addressed to Li-Hong Yang; lihongyang@hrbeu.edu.cn
}

Received 16 May 2013; Accepted 30 September 2013

Academic Editor: Rodrigo Lopez Pouso

Copyright ( 2013 Li-Hong Yang et al. This is an open access article distributed under the Creative Commons Attribution License, which permits unrestricted use, distribution, and reproduction in any medium, provided the original work is properly cited.

A numerical technique based on reproducing kernel methods for the exact solution of linear Volterra integral equations system of the second kind is given. The traditional reproducing kernel method requests that operator a satisfied linear operator equation $A u=f$, is bounded and its image space is the reproducing kernel space $W_{2}^{1}[a, b]$. It limits its application. Now, we modify the reproducing kernel method such that it can be more widely applicable. The $n$-term approximation solution obtained by the modified method is of high accuracy. The numerical example compared with other methods shows that the modified method is more efficient.

\section{Introduction}

The purpose of this paper is to solve a system of linear Volterra integral equations

$$
F(s)=G s+\int_{a}^{b} K(s, t) F(t) d t, \quad s \in[0,1],
$$

where

$$
\begin{aligned}
& F(s)=\left[f_{1}(s), f_{2}(s), \ldots, f_{n}(s)\right]^{T}, \\
& G(s)=\left[g_{1}(s), g_{2}(s), \ldots, g_{n}(s)\right]^{T}, \\
& K(s, t)=\left[k_{i, j}\right], \quad i, j=1,2, \ldots, n .
\end{aligned}
$$

In (1), the functions $K$ and $G$ are given, and $F$ is the solution to be determined. We assume that (1) has a unique solution. Volterra integral equation arises in many physical applications, for example, potential theory and Dirichlet problems, electrostatics, mathematical problems of radiative equilibrium, the particle transport problems of astrophysics and reactor theory, and radiative heat transfer problems [1-5]. Several valid methods for solving Volterra integral equation have been developed in recent years, including power series method [6], Adomain's decomposition method [7], homotopy perturbation method $[8,9]$, block by block method [10], and expansion method [11].
Since the reproducing kernel space $W_{2}^{1}[a, b]$, which is a special Hilbert space, is constructed in 1986 [12], the reproducing kernel theory has been applied successfully to many linear and nonlinear problems, such as differential equation, population model, and many other equations appearing in physics and engineering [12-21]. The traditional reproducing kernel method is limited, because it requires that the image space of operator $A$ in linear operator equation $A u=f$ is $W_{2}^{1}[a, b]$ and operator $A$ must be bounded. In order to enlarge its application range, the MRKM removes the boundedness of $A$ and weakens its image space to $L^{2}[a, b]$. Subsequently, we apply the MRKM to obtain the series expression of the exact solution for (1). The $n$-term approximation solution is provided by truncating the series. The final numerical comparisons between our method and other methods show the efficiency of the proposed method. It is worth to mention that the MRKM can be generalized to solve other system of linear equations.

\section{Preliminaries}

2.1. The Reproducing Kernel Space $W_{2}^{1}[0,1]$. The reproducing kernel space $W_{2}^{1}[0,1]$ consists of all absolute continuous realvalued functions, which defined on the closed interval $[0,1]$, and the first derivative functions belong to $L^{2}[0,1]$. 
The inner product and the norm are equipped with

$$
\begin{gathered}
(u, v)_{w_{2}^{1}}=u(0) v(0)+\int_{0}^{1} u^{\prime}(x) v^{\prime}(x) d x, \quad \forall u, v \in w_{2}^{1}, \\
\|u\|_{W_{2}^{1}}=\sqrt{(u, v)_{w_{2}^{1}}} .
\end{gathered}
$$

Theorem 1. $W_{2}^{1}[0,1]$ is a reproducing kernel space with reproducing kernel [22]

$$
R_{x}(y)= \begin{cases}1+y, & y \leq x \\ 1+x, & y>x\end{cases}
$$

that is, for every $x \in[0,1]$ and $u \in W_{2}^{1}$, it follows that

$$
\left(u(y), R_{x}(y)\right)_{w_{2}^{1}}=u(x) .
$$

2.2. The Reproducing Kernel Space $W_{2}^{2}[0,1]$. The reproducing kernel space $W_{2}^{2}[0,1]$ consists of all real-valued functions in which the first derivative functions are absolute continuous on the closed interval $[0,1]$ and the second derivative functions belong to $L^{2}[0,1]$.

The inner product and the norm are equipped with

$$
\begin{aligned}
(u, v)_{W_{2}^{2}}= & \sum_{k=0}^{1} u^{(k)}(0) v^{(k)}(0) \\
& +\int_{0}^{1} u^{\prime \prime}(x) v^{\prime \prime}(x) d x, \quad \forall u, v \in W_{2}^{2}[0,1] \\
\|u\|_{W_{2}^{2}}=\sqrt{(u, u)_{w_{2}^{2}}} &
\end{aligned}
$$

Theorem 2 . $W_{2}^{2}[0,1]$ is a reproducing kernel space with reproducing kernel [22]

$$
Q(x, y)= \begin{cases}1+x \times y+\frac{x \times y^{2}}{2}-\frac{y^{3}}{6}, & y \leq x \\ 1+x \times y+\frac{x^{2} \times y}{2}-\frac{x^{3}}{6}, & y>x ;\end{cases}
$$

that is, for every $x \in[0,1]$ and $u \in W_{2}^{2}$, it follows that

$$
(u(y), Q(x, y))_{w_{2}^{2}}=u(x) .
$$

The proof of Theorems 1 and 2 can be found in [23].

2.3. Hilbert Space E. Hilbert space $E$ is defined by

$$
E=\bigoplus_{i=1}^{n} W_{2}^{1}=\left\{\left(u_{1}, \ldots, u_{n}\right)^{T} \mid u_{i} \in w_{2}^{1}, i=1, \ldots, n\right\} .
$$

The inner product and the norm are given by

$$
\begin{gathered}
(u, v)_{E}=\sum_{i=1}^{n}\left(u_{i}, v_{i}\right)_{w_{2}^{1}}, \\
\|u\|_{E}=\sqrt{(u, u)_{E}} .
\end{gathered}
$$

It is easy to prove that $E$ is a Hilbert space.

\section{The Exact Solution of (1)}

3.1. Identical Transformation of (1). Consider the ith equation of (1):

$$
f_{i}(s)-\sum_{j=1}^{n} \int_{0}^{s} K_{i j}(s, t) f_{j}(t) d t=g_{i}(s) .
$$

Define operator $A_{i j}: W_{2}^{1} \rightarrow L^{2}[0,1], j=1, \ldots, n$,

$$
A_{i j}= \begin{cases}u(s)-\int_{0}^{1} k_{i j}(s, t) u(t) d t, & j=i \\ -\int_{0}^{s} k_{i j}(s, t) u(t) d t, & j \neq i,\end{cases}
$$

where $u \in W_{2}^{1}$. Then, (1) can be turned into

$$
\begin{gathered}
A_{11} f_{1}+A_{12} f_{2}+\cdots+A_{1 n} f_{1 n}=g_{1}(s) \\
A_{21} f_{1}+A_{22} f_{2}+\cdots+A_{2 n} f_{1 n}=g_{2}(s) \\
\vdots \\
A_{n 1} f_{1}+A_{n 2} f_{2}+\cdots+A_{n n} f_{1 n}=g_{n}(s),
\end{gathered}
$$

where $F(s)=\left[f_{1}(s), f_{2}(s), \ldots, f_{n}(s)\right]^{T} \in E$.

3.2. The Exact solution of (1). Let $\left\{x_{i}\right\}_{i=1}^{\infty}$ be a dense subset of interval $[0,1]$, and define

$$
\begin{gathered}
\Psi_{i j}(x)=\left(\left.A_{j_{1}, y} R_{x}(y)\right|_{y=x_{i}},\left.A_{j_{2}, y} R_{x}(y)\right|_{y=x_{i}}, \ldots,\right. \\
\left.\left.A_{j_{n}, y} R_{x}(y)\right|_{y=x_{i}}\right)^{T}
\end{gathered}
$$

for every $j=1,2, \ldots, n, i=1,2, \ldots$; the subscript $y$ of $A_{i j, y}$ means that the operator $A_{i j}$ acts on the function of $y$. It is easy to prove that $\Psi_{i j} \in E$.

Theorem 3. $\left\{\Psi_{i 1}, \Psi_{i 2}, \ldots, \Psi_{i n}\right\}_{i=1}^{\infty}$ is complete in $E$.

Proof. Take $u=\left(u_{1}, u_{2}, \ldots, u_{n}\right)^{T} \in E$ such that $\left(u(x), \Psi_{i j}(x)\right)$ $=0$ for every $j=1,2, \ldots, n, i=1,2, \ldots$.

From this fact, it holds that

$$
\left(u(x), \Psi_{i j}(x)\right)
$$

$$
=\left(\left(u_{1}, u_{2}, \ldots, u_{n}\right)^{T},\right.
$$

$$
\begin{aligned}
& \left(\left.A_{j 1, y} R_{x}(y)\right|_{y=x_{i}},\right. \\
& \left.\left.\left.\quad A_{j 2, y} R_{x}(y)\right|_{y=x_{i}}, \ldots,\left.A_{j n, y} R_{x}(y)\right|_{y=x_{i}}\right)^{T}\right) \\
= & \left.\sum_{k=1}^{n} A_{j k, y}\left(u_{k}(x), R_{x}(y)\right)_{w_{2}^{1}}\right|_{y=x_{i}} \\
= & \sum_{k=1}^{n} A_{j k} u_{k}\left(x_{i}\right)=0,
\end{aligned}
$$


for every $j=1,2, \ldots, n$. The dense $\left\{x_{i}\right\}_{i=1}^{\infty}$ assumes that

$$
\begin{array}{r}
A_{11} u_{1}+A_{12} u_{2}+\cdots+A_{1 n} u_{n}=0 \\
A_{21} u_{1}+A_{22} u_{2}+\cdots+A_{2 n} u_{n}=0 \\
\vdots \\
A_{n 1} u_{1}+A_{n 2} u_{2}+\cdots+A_{n n} u_{n}=0 .
\end{array}
$$

Since (16) has a unique solution, it follows that $u=$ $u_{1}, u_{2}, \ldots, u_{n}{ }^{T}=0$. This completes the proof.

We arrange $\Psi_{11}, \Psi_{12}, \ldots, \Psi_{1 n}, \Psi_{21}, \Psi_{22}, \ldots, \Psi_{2 n}, \ldots, \Psi_{i 1}, \Psi_{i 2}$, $\ldots, \Psi_{i n}, \ldots$, denoted by $\left\{r_{i}\right\}_{i=1}^{\infty}$; that is, $r_{1}=\Psi_{11}, r_{2}=\Psi_{12}$, $\ldots, r_{n}=\Psi_{1 n}, r_{n+1}=\Psi_{21}, r_{n+2}=\Psi_{22}, \ldots, r_{n+n}=\Psi_{2 n}, \ldots$. In a general way, $r_{(i-1) n+j}=\Psi_{i j}, i=1,2,3, \ldots ; j=1$, $2, \ldots, n$. The orthogonal basis $\left\{\bar{r}_{i}\right\}_{i=1}^{\infty}$ in $E$ from Gram-Schmidt orthogonalization of $\left\{r_{i}\right\}_{i=1}^{\infty}$ is as follows:

$$
\bar{r}_{i}=\sum_{k=1}^{i} \beta_{i k} r_{k}, \quad i=1,2, \ldots
$$

Theorem 4. The exact solution of (1) can be expressed by

$$
F(x)=\sum_{i=1}^{\infty} \sum_{k=1}^{i} \beta_{i k} \rho_{k} \bar{r}_{i}(x)
$$

where $\rho_{k}=\left(F(x), r_{k}\right)_{E}$; if $r_{k}=\Psi_{j l}$, then $\rho_{k}=g_{l}\left(x_{j}\right)$.

Proof. Assume that $F(x)$ is the exact solution of (1). $F(x)$ can be expanded to Fourier series in terms of normal orthogonal basis $\left\{\bar{r}_{i}(x)\right\}_{i=1}^{\infty}$ in $E$ :

$$
F(x)=\sum_{i=1}^{\infty}\left(F, \bar{r}_{i}\right)_{E} \bar{r}_{i}(x)=\sum_{i=1}^{\infty} \sum_{k=1}^{i} \beta_{i k}\left(F, r_{k}\right)_{E} \bar{r}_{i}(x) ;
$$

if $\rho_{k}=\left(F, r_{k}\right)_{E}$, then

$$
F(x)=\sum_{i=1}^{\infty} \sum_{k=1}^{i} \beta_{i j} \rho_{k} \bar{r}_{i}(x)
$$

When $r_{k}=\Psi_{j l}$, it holds that

$$
\rho_{k}=\left(F, \Psi_{j l}\right)=\sum_{k=1}^{n} A_{l k} u_{k}\left(x_{j}\right)=g_{l}\left(x_{j}\right) .
$$

Corollary 5. The approximate solution of (1) is

$$
F_{m}(x)=\sum_{i=1}^{m} \sum_{k=1}^{i} \beta_{i k} \rho_{k} \bar{r}_{i}(x)=\left(f_{1, m}, f_{2, m}, \ldots, f_{n, m}\right)^{T},
$$

and $f_{i, m}(x)$ converges uniformly to $f_{i}(x)$ on $[0,1]$ as $m \rightarrow \infty$ for every $i=1,2, \ldots, n$.

Proof. Obviously, $\left\|F_{m}-F\right\|_{E}^{2} \rightarrow 0$ holds as $m \rightarrow \infty$; that is, $F_{m}(x)$ is the approximate solution of (1).
Note that $\sum_{i=1}^{n}\left\|f_{i, m}-f_{i}\right\|_{W_{2}^{1}}^{2}=\left\|F_{m}-F\right\|_{E}^{2} \rightarrow 0$. Combining with the expression of $R_{x}(y)$, we have

$$
\begin{aligned}
\left|f_{i, m}-f_{i}\right| & =\left|\left(f_{i, m}(y)-f_{i}(y), R_{x}(y)\right)_{W_{2}^{1}}\right| \\
& \leq\left\|f_{i, m}-f_{i}\right\|_{W_{2}^{1}} \cdot\left\|R_{x}(y)\right\|_{W_{2}^{1}} \\
& =\left\|f_{i, m}-f_{i}\right\|_{W_{2}^{1}} \sqrt{R_{x}(x)} \\
& \leq \sqrt{2}\left\|f_{i, m}-f_{i}\right\|_{W_{2}^{1}}, \quad \forall x \in[0,1] .
\end{aligned}
$$

It shows that $f_{i, m}$ converges uniformly to $f_{i}$ on $[0,1]$ as $m \rightarrow$ $\infty$ for every $i=1,2, \ldots, n$. So the proof is complete.

Remark 6. If $k_{i j}(s, t) \in C([0,1] \times[0,1])$ and $g_{i} \in W_{2}^{2}$ in (1), then it is reasonable to regard the unknown functions as the elements of $W_{2}^{2}$.

\section{Numerical Examples}

Taking nodes $\left\{x_{i}=(i-1) /(N-1)\right\}_{i=1}^{N}, f_{i, N}$ is the approximate solutions of $f_{i}$, and $e\left(f_{i, N}\right)$ denotes the absolute errors of $f_{i}, i=1,2, \ldots, n$. According to Remark 6, we solve the following two examples appearing in [11] in $W_{2}^{2}$.

Example 7. Consider the following system of Volterra integral equations of the second kind [11]:

$$
\begin{gathered}
f_{1}(s)=g_{1}(s)+\int_{0}^{s}(s-t)^{3} f_{1}(t) d t+\int_{0}^{s}(s-t)^{2} f_{2}(t) d t \\
f_{2}(s)=g_{2}(s)+\int_{0}^{s}(s-t)^{4} f_{1}(t) d t \\
+\int_{0}^{s}(s-t)^{3} f_{2}(t) d t,
\end{gathered}
$$

where $g_{1}(s)$ and $g_{2}(s)$ are chosen such that the exact solution is $f_{1}(s)=1+s^{2}, f_{2}(s)=1+s-s^{3}$. The numerical results obtained by using the present method are compared with [11] in Table 1.

Example 8. Consider the following system of linear Volterra integral equations of the second kind [11]:

$$
\begin{gathered}
f_{1}(s)=g_{1}(s)+\int_{0}^{s}(\sin (s-t)-1) f_{1}(t) d t \\
+\int_{0}^{s}(1-t \cos s) f_{2}(t) d t \\
f_{2}(s)=g_{2}(s)+\int_{0}^{s}\left(f_{1}(t)\right) d t+\int_{0}^{s}(s-t) f_{2}(t) d t
\end{gathered}
$$

where $g_{1}(s)$ and $g_{2}(s)$ are chosen such that the exact solution is $f_{1}(s)=\cos s, f_{2}(s)=\sin s$. The numerical results obtained by using the present method are compared with [11] in Table 2. 
TABLE 1: Absolute errors for Example 7.

\begin{tabular}{lcccc}
\hline Nodes $x_{i}$ & Errors $e\left(f_{1}\right)[11]$ & Errors $e\left(f_{1,100}\right)$ & Errors $e\left(f_{2}\right)[11]$ & Errors $e\left(f_{2,100}\right)[11]$ \\
\hline 0.0 & 0 & $1.58309 E-10$ & 0 & $3.98245 E-10$ \\
0.1 & $2.63472 E-7$ & $3.92220 E-12$ & $2.11685 E-8$ & $3.94493 E-10$ \\
0.2 & $1.62592 E-5$ & $3.21563 E-10$ & $2.61132 E-6$ & $4.72710 E-10$ \\
0.3 & $1.74905 E-4$ & $5.95890 E-10$ & $4.18979 E-5$ & $6.19366 E-10$ \\
0.4 & $8.93799 E-4$ & $5.11051 E-10$ & $2.86285 E-4$ & $6.95422 E-10$ \\
0.5 & $3.00491 E-3$ & $2.46104 E-10$ & $1.19940 E-3$ & $4.19959 E-10$ \\
0.6 & $7.47528 E-3$ & $1.98685 E-9$ & $3.56141 E-3$ & $5.90035 E-10$ \\
0.7 & $1.40733 E-2$ & $5.01512 E-9$ & $7.74239 E-3$ & $2.83080 E-9$ \\
0.8 & $1.78384 E-2$ & $9.62848 E-9$ & $1.09171 E-2$ & $6.94058 E-9$ \\
0.9 & $4.97756 E-3$ & $1.61180 E-8$ & $2.27326 E-3$ & $1.36984 E-8$ \\
1.0 & $3.84378 E-2$ & $2.49043 E-8$ & $3.32111 E-2$ & $2.42565 E-8$ \\
\hline
\end{tabular}

TABLE 2: Absolute errors for Example 8.

\begin{tabular}{lcccc}
\hline Nodes $x_{i}$ & Errors $e\left(f_{1}\right)[11]$ & Errors $e\left(f_{1,100}\right)$ & Errors $e\left(f_{2}\right)[11]$ & Errors $e\left(f_{2,100}\right)[11]$ \\
\hline 0.0 & 0 & $6.93348 E-11$ & 0 & $3.60316 E-11$ \\
0.1 & $1.37735 E-4$ & $4.53518 E-09$ & $1.52721 E-4$ & $2.75123 E-08$ \\
0.2 & $9.27188 E-4$ & $8.84879 E-09$ & $1.14715 E-3$ & $3.10611 E-08$ \\
0.3 & $2.67117 E-3$ & $1.28253 E-08$ & $3.71248 E-3$ & $3.53307 E-08$ \\
0.4 & $5.45507 E-3$ & $1.65442 E-08$ & $8.57201 E-3$ & $4.03402 E-08$ \\
0.5 & $9.22670 E-3$ & $2.00881 E-08$ & $1.64412 E-2$ & $4.61209 E-08$ \\
0.6 & $1.38644 E-2$ & $2.35657 E-09$ & $2.78243 E-2$ & $5.27214 E-08$ \\
0.7 & $1.92960 E-2$ & $2.71160 E-08$ & $4.25337 E-2$ & $6.02041 E-08$ \\
0.8 & $2.56349 E-2$ & $3.09302 E-08$ & $5.91212 E-2$ & $6.86601 E-08$ \\
0.9 & $3.31574 E-2$ & $3.52645 E-08$ & $7.48883 E-2$ & $7.82029 E-08$ \\
1.0 & $4.19808 E-2$ & $3.67322 E-08$ & $8.70896 E-2$ & $1.02387 E-07$ \\
\hline
\end{tabular}

\section{Conclusion}

In this paper, we modify the traditional reproducing kernel method to enlarge its application range. The new method named MRKM is applied successfully to solve a system of linear Volterra integral equations. The numerical results show that our method is effective. It is worth to be pointed out that the MRKM is still suitable for solving other systems of linear equations.

\section{Acknowledgments}

The research was supported by the Fundamental Research Funds for the Central Universities.

\section{References}

[1] Y. Ren, B. Zhang, and H. Qiao, "A simple Taylor-series expansion method for a class of second kind integral equations," Journal of Computational and Applied Mathematics, vol. 110, no. 1, pp. 15-24, 1999.

[2] K. Maleknejad and Y. Mahmoudi, "Numerical solution of linear Fredholm integral equation by using hybrid Taylor and blockpulse functions," Applied Mathematics and Computation, vol. 149, no. 3, pp. 799-806, 2004.

[3] S. Yalçınbaş and M. Sezer, "The approximate solution of highorder linear Volterra-Fredholm integro-differential equations in terms of Taylor polynomials," Applied Mathematics and Computation, vol. 112, no. 2-3, pp. 291-308, 2000.

[4] S. Yalçinbaş, "Taylor polynomial solutions of nonlinear Volterra-Fredholm integral equations," Applied Mathematics and Computation, vol. 127, no. 2-3, pp. 195-206, 2002.

[5] E. Deeba, S. A. Khuri, and S. Xie, "An algorithm for solving a nonlinear integro-differential equation," Applied Mathematics and Computation, vol. 115, no. 2-3, pp. 123-131, 2000.

[6] A. Tahmasbi and O. S. Fard, "Numerical solution of linear Volterra integral equations system of the second kind," Applied Mathematics and Computation, vol. 201, no. 1-2, pp. 547-552, 2008.

[7] E. Babolian, J. Biazar, and A. R. Vahidi, "On the decomposition method for system of linear equations and system of linear Volterra integral equations," Applied Mathematics and Computation, vol. 147, no. 1, pp. 19-27, 2004.

[8] J. Biazar and H. Ghazvini, "He's homotopy perturbation method for solving systems of Volterra integral equations of the second kind," Chaos, Solitons \& Fractals, vol. 39, no. 2, pp. 770-777, 2009.

[9] E. Yusufoğlu (Agadjanov), "A homotopy perturbation algorithm to solve a system of Fredholm-Volterra type integral equations," Mathematical and Computer Modelling, vol. 47, no. 11-12, pp. 1099-1107, 2008.

[10] R. Katani and S. Shahmorad, "Block by block method for the systems of nonlinear Volterra integral equations," Applied Mathematical Modelling, vol. 34, no. 2, pp. 400-406, 2010. 
[11] M. Rabbani, K. Maleknejad, and N. Aghazadeh, "Numerical computational solution of the Volterra integral equations system of the second kind by using an expansion method," Applied Mathematics and Computation, vol. 187, no. 2, pp. 1143-1146, 2007.

[12] M. G. Cui and Z.X. Deng, "On the best operator of interpolation in $W_{2}^{1}[a, b]$," Mathematica Numerica Sinica, vol. 2, pp. 209-216, 1986.

[13] M. Cui and F. Geng, "A computational method for solving onedimensional variable-coefficient Burgers equation," Applied Mathematics and Computation, vol. 188, no. 2, pp. 1389-1401, 2007.

[14] M. Cui and F. Geng, "A computational method for solving thirdorder singularly perturbed boundary-value problems," Applied Mathematics and Computation, vol. 198, no. 2, pp. 896-903, 2008.

[15] M. Cui and Z. Chen, "The exact solution of nonlinear agestructured population model," Nonlinear Analysis. Real World Applications, vol. 8, no. 4, pp. 1096-1112, 2007.

[16] F. Z. Geng and M. G. Cui, "Solving singular nonlinear two-point boundary value problems in the reproducing kernel space," Journal of the Korean Mathematical Society, vol. 45, no. 3, pp. 631-644, 2008.

[17] M. G. Cui and Y. B. Yan, "The representation of the solution of a kind operator equation $A u=f$," Journal of Chinese Universities, vol. 3, pp. 82-86, 1995.

[18] O. Abu Arqub, M. Al-Smadi, and S. Momani, "Application of reproducing kernel method for solving nonlinear FredholmVolterra integrodifferential equations," Abstract and Applied Analysis, vol. 2012, Article ID 839836, 16 pages, 2012.

[19] O. A. Arqub, M. Al-Smadi, and N. Shawagfeh, "Solving Fredholm integro-differential equations using reproducing kernel Hilbert space method," Applied Mathematics and Computation, vol. 219, no. 17, pp. 8938-8948, 2013.

[20] M. Al-Smadi, O. Abu Arqub, and S. Momani, "A computational method for two-point boundary value problems of fourth-order mixed integrodifferential equations," Mathematical Problems in Engineering, vol. 2013, Article ID 832074, 10 pages, 2013.

[21] M. Al-Smadi, O. Abu Arqub, and N. Shawagfeh, "Approximate solution of BVPs for 4th-order IDEs by using RKHS method," Applied Mathematical Sciences, vol. 6, no. 49-52, pp. 2453-2464, 2012.

[22] Z. Chen and Y. Lin, "The exact solution of a linear integral equation with weakly singular kernel," Journal of Mathematical Analysis and Applications, vol. 344, no. 2, pp. 726-734, 2008.

[23] M. Cui and Y. Lin, Nonlinear Numerical Analysis in the Reproducing Kernel Space, Nova Science Publishers, New York, NY, USA, 2009. 


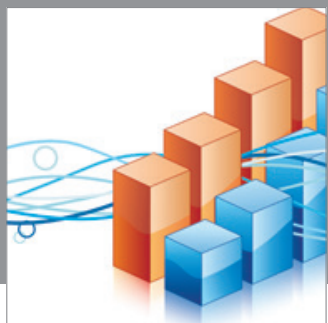

Advances in

Operations Research

mansans

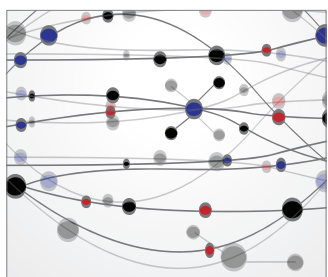

The Scientific World Journal
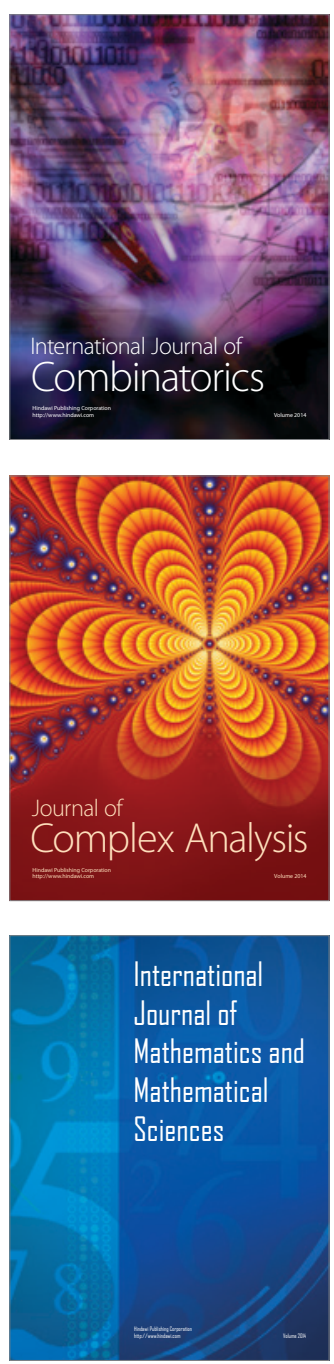
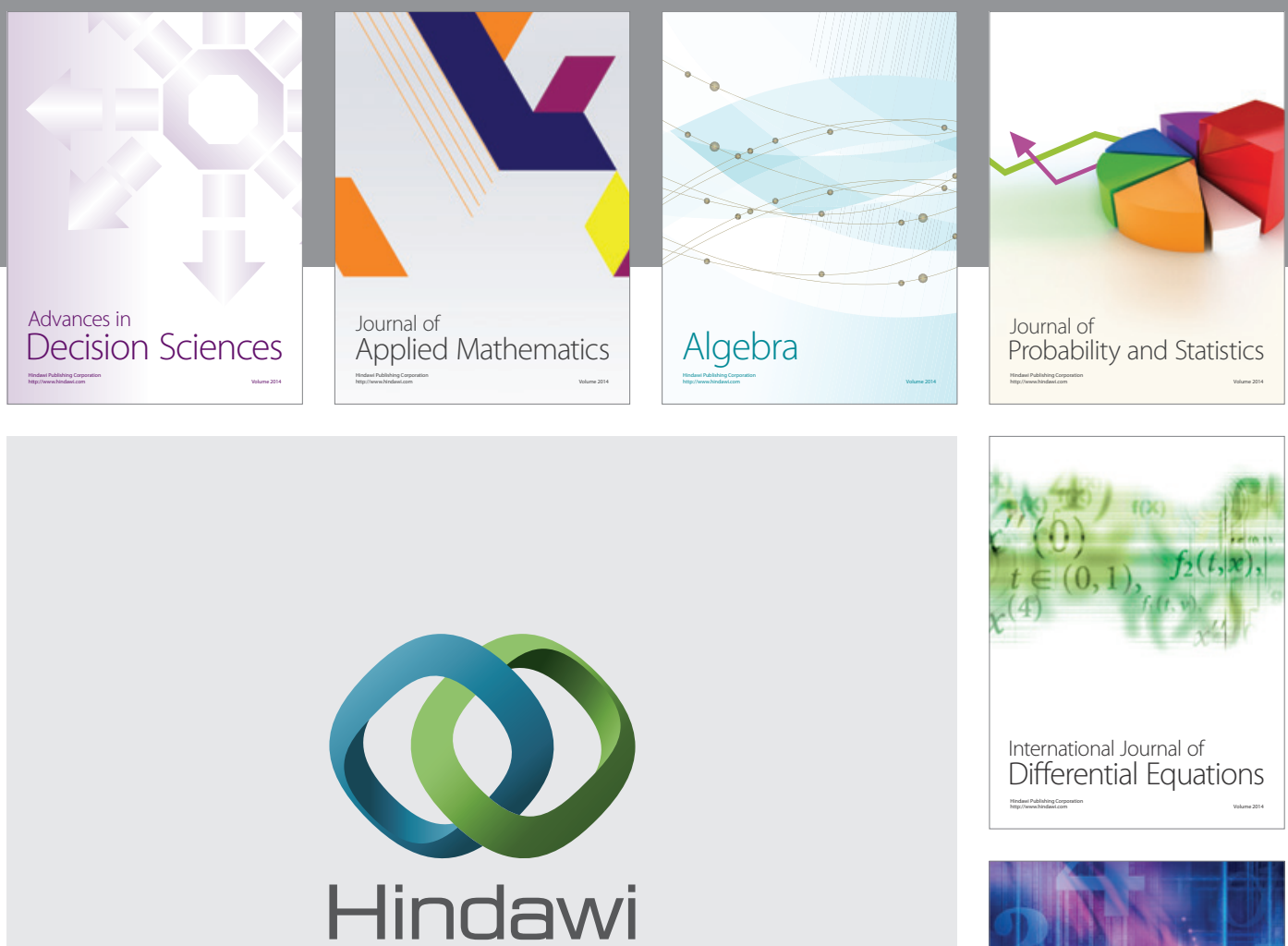

Submit your manuscripts at http://www.hindawi.com
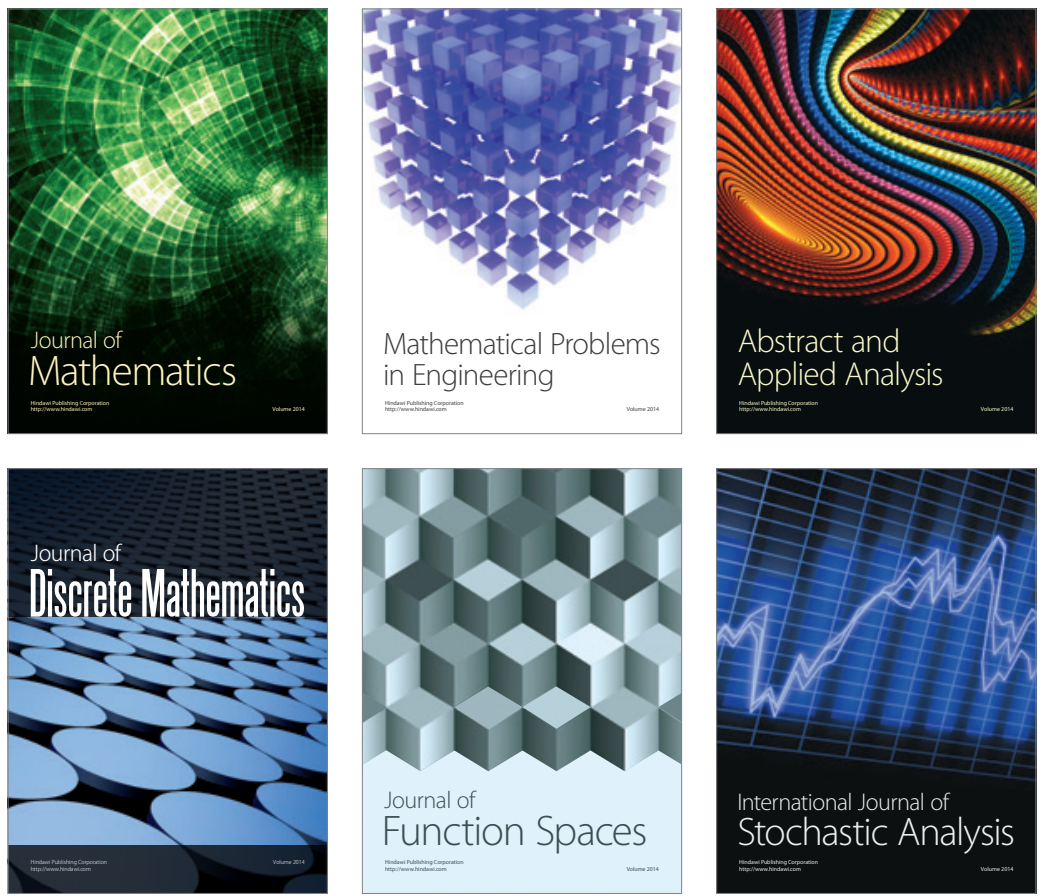

Journal of

Function Spaces

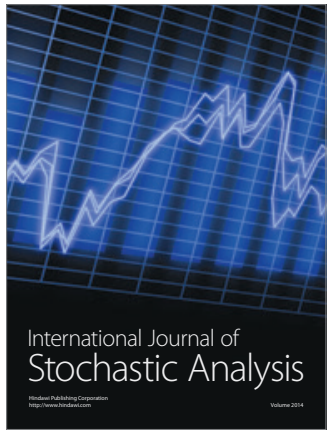

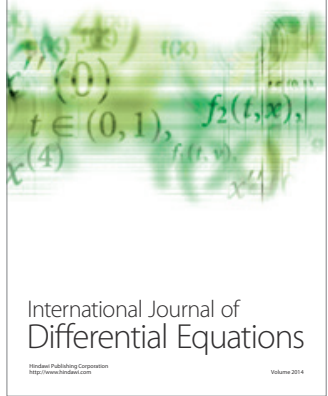
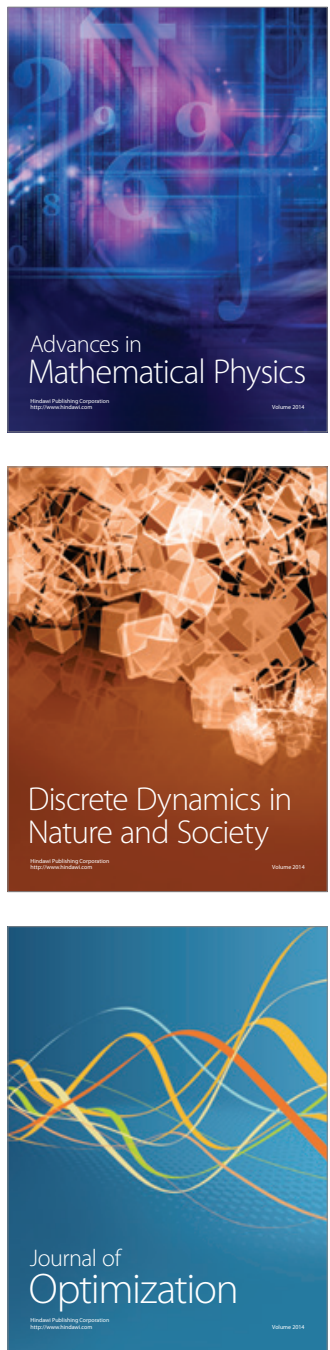